\title{
MHD Flow-Control for Hypersonic Flight
}

\author{
J.P. Petit and J. Geffray \\ LAMBDA Laboratory, France*
}

\begin{abstract}
At the beginning of the seventies, experimental research using short duration supersonic shock driven wind tunnel were conducted and showed the efficiency of Lorentz force action on supersonic (Mach 1.4) dense (1 bar) low magnetic Reynolds number hot $(10000 \mathrm{~K})$ argon flows. When working as a generator, the linear Faraday MHD converter was efficient enough, due to high electrical conductivity $(3000 \mathrm{~S} / \mathrm{m})$ to create a front shock wave at the intake of the constant cross-section MHD converter. Accelerations up to $5000 \mathrm{~m} / \mathrm{s}$ were obtained in $10 \mathrm{~cm}$ long channels. This suggested the possibility to achieve complete shock wave and turbulent wake cancellation, through MHD bypass concept. Such work gave publications in peer-reviewed journals and presentation in international MHD meetings (French Academy of Sciences 1970, Moscow 1983, Tsukuba 1986, Beijing 1992). The work was conducted both through computational simulations (based on the method of characteristics) and hydraulic simulation experiments. We present the synthesis of such studies through a project of a hypersonic vehicle based on an MHD bypass concept, landing under its own steam, using classical turbojets. Then, at Mach 3 and high altitude, MHD controlled inlets are opened. A wall converter slows down the hypersonic incoming air flow, without excessive heating, feeding a ramjet system. The subsequent electric power provides an additional impulse to the flow in the exhaust section. Cruise Mach number: 12. Local Hall parameter regime produces high voltage that, sent to the leading edges, creates a plasma cushion which prevents too high thermal flux. Lift is provided by wave riding technique. Additional rocket propeller could transform it as a reusable space launcher.
\end{abstract}

PACS numbers: 02.30.Jr, 47.40.Ki, 47.65.-d, 47.85.lb, 52.30.Cv, 52.75.Fk

\section{Introduction}

Fluid dynamics specialists know that shock waves take place where the characteristics of the Navier-Stokes equations system, the Mach surfaces, accumulate. It is possible to modify the local value of the Mach number, either by changing the flow temperature, i.e. the local value of the sound velocity, or by changing the flow velocity. As an example, Fig. 1a, b shows a schematic view of a two-dimensional Mach lines pattern, modified by slowing down or accelerating the gas in a Faraday MHD converter, through the Lorentz force action.

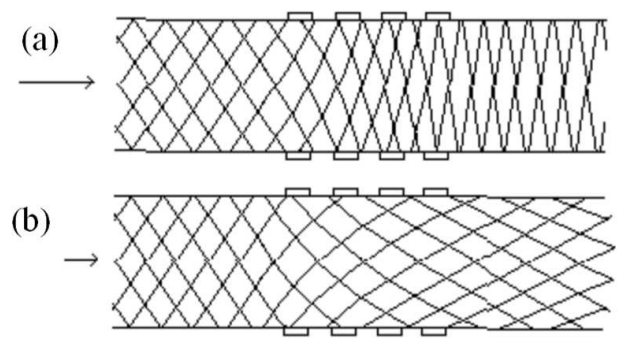

Fig. 1. (a) Slowing down, (b) accelerating.

In the first diagram, if the action is strong enough, the characteristics become tangent and a front shock forms, as experienced in lab, in MHD shock tube experiments

\footnotetext{
* www.mhdprospects.com/lambda/
}

in 1965 [1]. Next (Fig. 2a and b) the 2D characteristics are given, as computed from the Navier-Stokes equations around some sort of flat wing.
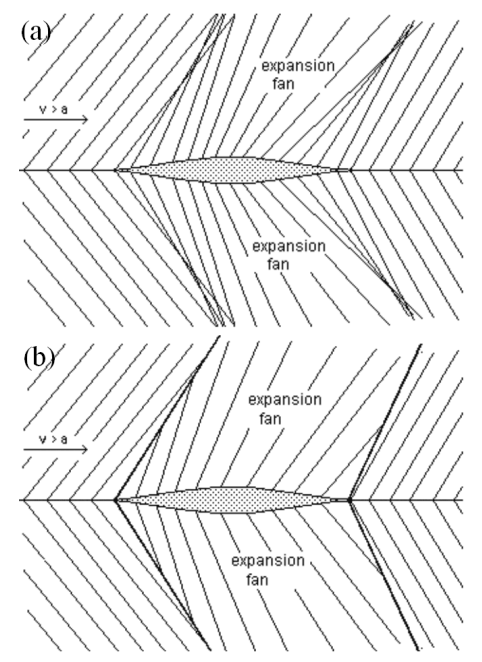

Fig. 2. (a) Characteristic curves (Mach lines), (b) plane shock waves.

Is it possible to cancel the shock waves around a body moving in a gas at supersonic speed? Yes, if the characteristic lines are prevented from intersecting each others. The fluid needs to be accelerated where the shock waves tend to form, and slowed down in the expansion regions. 
One can say differently: the parallelism of this family of characteristics must be kept around the body. This was first demonstrated by hydraulic analogy experiments in 1976. Then the complete cancellation of the shock wave system was calculated in a Ph.D. thesis [2], corresponding to planned experiments in a shock driven wind tunnel (argon at 1 bar, $10000 \mathrm{~K}$ ). We needed a transverse magnetic field and three pairs of electrodes. Then the characteristics pattern was as shown in Fig. 3.

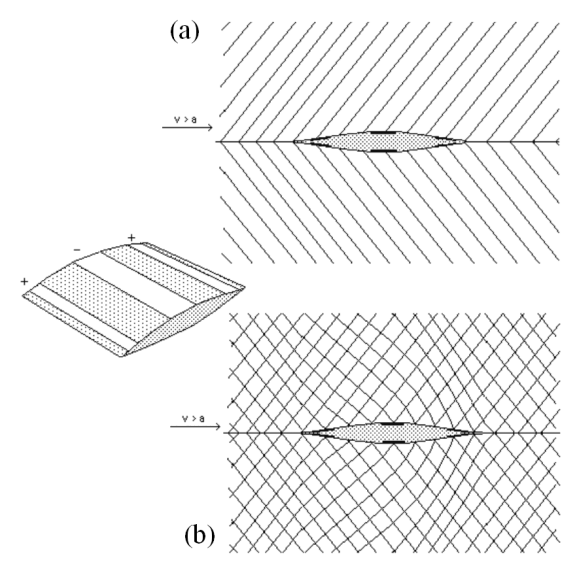

Fig. 3. Shock wave cancellation around a flat wing, due to suitable $J \times B$ force field.

Let us notice this introduced the concept of MHD bypass. Details are given in [3]. Unfortunately, the lack of funding at that time did not allow the job to be finished and the experiment to be performed. But now, twenty years later, a new funding makes possible to restart the project on new basis.

A lot of additional experiments are planned, in short duration hot flows (shock driven wind tunnel) and short duration cold flows. In the first the ionization comes from the high gas temperature (argon $10000 \mathrm{~K}$ ). In the second we will use wall ionizers or microwave ionization. Among the different purchased goals is a project of hypersonic MHD-driven inlet, coupled to acceleration at exhaust, achieving a MHD bypass system.

\section{Applications to hypersonic planes}

The working principle is described in the following diagrams. The conception of a hypersonic vehicle, cruising in the Mach 12-15 range at high altitude, implies resolving two fundamental problems:

- To handle air compression behind shock wave into the ramjet inlet, so the temperature does not reach a value causing a thermal ablation of the material.

- To protect the leading edges.

The MHD bypass concept is quite simple. An MHD converter is a reversible device, so the same machine can act either as an electrical generator, or as a fluid accelerator. A turbojet already achieves some sort of mechanical bypass, since a part of the energy given to the gas into the combustion chamber is used to rotate the engine axis through a turbine, but this energy is also transmitted upstream, where it is used to compress the air or even to activate a fan. In an MHD bypass system, the front of the vehicle works as an MHD generator. MHD bypass for hypersonic planes has been actively studied for example in the USSR with the Ayaks project publicly known only since 1996 [4-8]. Some scientists estimated that on-board MHD converters could be of the Faraday type, but the geometry of the generator proposed in this paper (Fig. 4a and b) corresponds rather to a top wall converter with many electrodes close together, as described in 1983 in Ref. [1] where it was presented in its accelerator version.
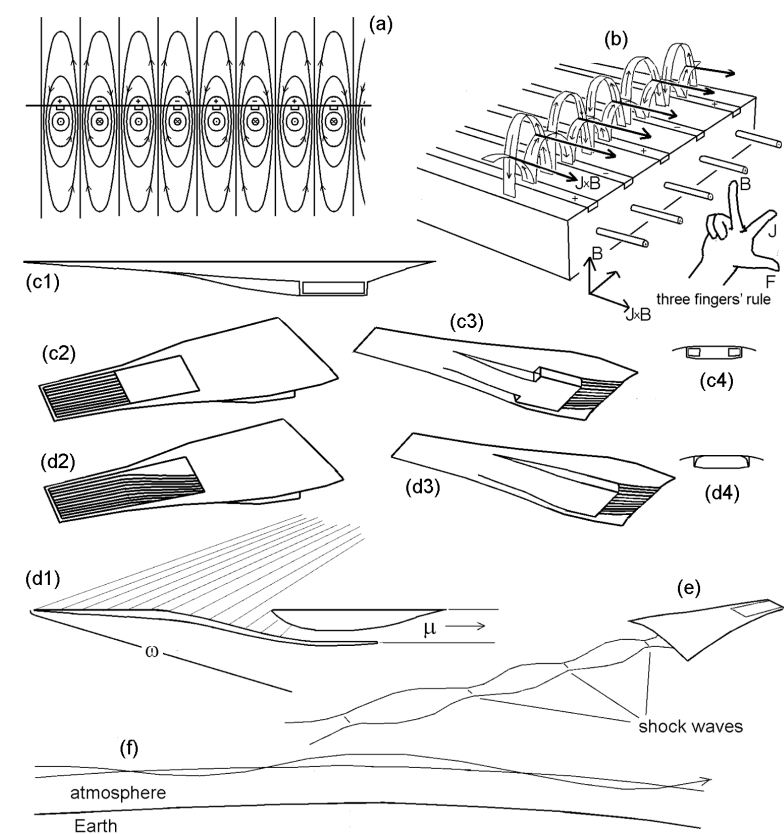

Fig. 4. MHD-bypass with wall convertors: configuration at different altitudes and speeds.

The induced electric field is $V \times B$. The point of this wall converter is the very short distance between electrodes. In thin air at altitudes around 150000 feet, the high electrical conductivity allows the MHD converter to work in power generation mode. The electric power collected by this front wall converter is sent to a second wall converter located at the rear, working there as an accelerator of the exhaust gas. As indicated in the diagrams, the vehicle has two configuration types. In Fig. 4c1 to c4) it is a highly-swept wing aircraft propelled by conventional turbojets, which takes off under its own steam. Classically, a variable exhaust nozzle allows the gas relaxation ratio to be adapted according to altitude, therefore to the surrounding pressure, but it is not possible here. Therefore the design of the nozzle is half-guided (on its upper part, the bottom part being in free-surface flow) and corresponds to the pressure for a high altitude cruise. After take-off and while climbing through the sky below its optimum altitude, such a noz- 
zle does not work properly, like the nozzles of the first jet engines which ejected an over-extended gas: the exhaust gas looked like a series of dense regions (shocks) and rarefied sections (Fig. 4e). So while climbing, such a plane would leave a dotted contrail (donuts on a rope) which would let observers wrongly think about a pulsated operating mode. When the reached altitude is high enough for the MHD converter to work, turbojet air intakes are blocked out, and the intakes of two side-ramjets (Fig. 4c1 to c4) are linked up with the opened upper part of the aircraft, which is dotted with parallel wall electrodes, elements of the MHD wall generator. The electrical power generation is made to the detriment of the airflow velocity, which slows down progressively without creating a shock wave. The Fig. 4c2 evokes the characteristics straightening up near the wall, a key element we will study in our new lab. Thus the kinetic energy of the incoming air is only partially converted into thermal energy, which prevents it from heating too much till the ramjet intake. The Hall effect provides a high voltage axial component which, sent on the aircraft leading edge, generates a protective plasma cushion. The shock wave establishing underneath provides lift (wave rider design). The cruise alternates propelled stages and ballistic trajectory stretches (Fig. 4f). A circumterrestrial flight would be possible in a few hours. Using liquefied gas as a fuel, this aircraft could also be put into orbit. The addition of liquid oxygen into auxiliary rocket engines would complete the operation. The vehicle would act as a new space launcher, a priori more interesting than classical rockets, since it leans on air most of the time during the climbing.

\section{References}

[1] J.P. Petit, Le Mur du Silence, Berlin, 1983. Also available freely in English, Lithuanian, German, Russian, Polish, Spanish, Croatian, Serbian, Romanian at: http://www.savoir-sans-frontieres.com .

[2] B. Lebrun, Ph. D. Thesis, Aix Marseille University, 1987. See: mhdprospects.com .

[3] J.P. Petit, B. Lebrun, in: Proc. 9th Int. Conf. on MHD Electrical Power Generation, Tsukuba, Japan (Nov. 1986), Proc. III, Part 14.E - MHD Flow, art. 5, p. 1359.

[4] Project Ayax, St. Petersburg Gazette 121 (July 6, 2006).

[5] Patent RU 1803595, A scramjet and its mode of operation (Jan. 8, 1996).

[6] Patent RU 2138668, Air-breathing magnetogasdynamic engine (Feb. 24, 1998).

[7] E.P. Gurijanov, P.T. Harsha, in: Proc. 7th Int. Space Planes and Hypersonic Systems and Technologies Conf., Norfolk, VA (Nov. 1996).

[8] V.L. Fraishtadt, A.L. Kuranov, E.G. Sheikin, JTF, B 68, 43 (1998). 